\title{
ON THE STRONG LAW OF LARGE NUMBERS IN BANACH SPACES
}

\author{
ANANT P. GODBOLE
}

\begin{abstract}
We study the relationship between the geometry of a real separable Banach space $B$ (as manifested in its cotype, type, or logtype) and necessary or sufficient criteria for the validity of the Strong Law of Large Numbers (SLLN) for independent $B$-valued random variables, formulated in terms of the validity of a (verifiable) SLLN for real-valued random variables. Our results are the best possible of their kind and may be used in situations where the SLLN's of Hoffman-Jørgensen and Pisier, and Kuelbs and Zinn are inconclusive.
\end{abstract}

1. Introduction. Consider a sequence $\left\{X_{n}\right\}$ of independent symmetric random variables taking values in the real separable Banach space $(B,\|\cdot\|) .\left\{\varepsilon_{n}\right\}$ will denote an independent sequence of i.i.d. Rademacher random variables $\left(P\left(\varepsilon_{n}= \pm 1\right)=\right.$ $1 / 2, n \geq 1) .\left\{X_{n}\right\}$ is said to satisfy the strong (weak) law of large numbers $\left(\left\{X_{n}\right\} \in\right.$ SLLN (WLLN)) if $\left\|S_{n}\right\|=o(n)$ a.s. $\left(\left\|S_{n}\right\| / n \stackrel{\operatorname{Pr}}{\longrightarrow} 0\right)$, where $S_{n}=\sum_{j=1}^{n} X_{j}$. In [4] type $p$ spaces $(1 \leq p \leq 2)$ were characterized as those which the condition $\sum_{j=i}^{\infty} E\left\|X_{j}\right\|^{p} / j^{p}<\infty$ implies the SLLN. A SLLN without geometric restrictions on $B$ was proved in [1]. This theorem, though the best possible, is a consequence of the trivial fact that $\left\{X_{n}\right\} \in$ SLLN if $\left\{\left\|X_{n}\right\|\right\} \in \operatorname{SLLN}$ (see [2] for details). Clearly, therefore, moment or norm assumptions will not yield useful SLLN's in general unless additional assumptions are made. Kuelbs and Zinn [5] hypothesize, for example, that $\left\{X_{n}\right\} \in \mathrm{WLLN}$ and prove that $\left\{X_{n}\right\} \in \mathrm{SLLN}$ if $\left\|X_{j}\right\| \leq C j / L L j$ and $\sum_{n=1}^{\infty} \exp (-\varepsilon / \Lambda(n, 2))<\infty$ for each $\varepsilon>0$ (Theorem 2), or if $\sum_{n=1}^{\infty} \Lambda(n, p)^{r}<\infty$ for some $p \in[1,2]$ and $r \in(0, \infty)$ (Theorem 1) (where $L L n=\max (1, \log (\log n)$ ), $\Lambda(n, p)=1 / 2^{n p} \sum_{j \in I(n)} E\left\|X_{j}\right\|^{p}$, and $I(n)=\left\{2^{n}+1, \ldots, 2^{n+1}\right\}$. For $B=\mathbf{R}$ the conditions in the first result above are also necessary [9].

Our results are expressed in terms of the validity of a SLLN for real random variables. (The latter can be verified for an arbitrary stabilizing sequence using the criteria in $[8,11]$.) For example, we define logtype $p$ spaces $(1 \leq p \leq 2)$ as those in which there is a constant $C$ such that

$$
E\left\|\sum_{j=1}^{n} \varepsilon_{j} x_{j}\right\|^{p} \leq C(L L n)^{p-1} \sum_{j=1}^{n}\left\|x_{j}\right\|^{p} \quad \text { for each }\left\{x_{j}\right\}_{j=1}^{n} \text { in } B,
$$

and show that these are precisely the spaces in which the condition

$$
(L L n)^{p-1} / n^{p} \sum_{j=1}^{n}\left\|X_{j}\right\|^{p} \rightarrow 0 \quad \text { a.s. }
$$

Received by the editors April 2, 1986.

1980 Mathematics Subject Classification (1985 Revision). Primary 60B12; Secondary 46B20.

Key words and phrases. Type $p$ and cotype $q$ Banach spaces, Strong Law of Large Numbers. 
implies the SLLN. We show that our results (i) are the best of their kind, (ii) are conclusive in cases when most of the above-mentioned SLLN's are not, and (iii) can often be reexpressed in terms of the moments of the individual summands. Nonsymmetric random variables can be included in our discussion by a result of Kuelbs and Zinn [5]: $\left\{X_{n}\right\} \in \operatorname{SLLN}$ iff $\left\{X_{n}^{s}\right\} \in \operatorname{SLLN}$ and $\left\{X_{n}\right\} \in$ WLLN, where $X_{n}^{s}\left(\omega, \omega^{\prime}\right)=X_{n}(\omega)-X_{n}^{\prime}\left(\omega^{\prime}\right)$ is the symmetrized version of $X_{n}$, and $X_{n}^{\prime}$ is an independent copy of $X_{n}$.

\section{Necessary conditions.}

THEOREM 1. $B$ is of cotype $q(2 \leq q<\infty)$ iff each sequence $\left\{X_{n}\right\}$ of independent symmetric $B$-valued random variables satisfying the SLLN also satisfies $\sum_{j=1}^{n}\left\|X_{j}\right\|^{q} / n^{q} \rightarrow 0$ a.s.

ProOF. Let $B$ be of cotype $q$ and suppose $\left\{X_{n}\right\} \in$ SLLN. It follows that $\left\{\varepsilon_{n} x_{n}\right\}=\left\{\varepsilon_{n} X_{n}(\omega)\right\} \in$ SLLN for almost all $\omega$. As in [4], $E\left\|\sum_{j=1}^{n} \varepsilon_{j} x_{j}\right\|^{q} / n^{q} \rightarrow 0$ which by the cotype $q$ inequality shows that $\sum_{j=1}^{n}\left\|x_{j}\right\|^{q} / n^{q} \rightarrow 0$, as required. Conversely, let $\left\{x_{n}\right\} \subseteq B$ and set $X_{n}=n \varepsilon_{n} x_{n}(n \geq 1)$. By assumption,

$$
\sum_{j=1}^{n} j^{q}\left\|x_{j}\right\|^{q} / n^{q} \rightarrow 0 \text { if } \sum_{j=1}^{n} j \varepsilon_{j} x_{j} / n \rightarrow 0 \quad \text { a.s. }
$$

and thus (by Kronecker's Lemma) if $\sum_{j=1}^{\infty} \varepsilon_{j} x_{j}$ converges in $L^{q}(B)$. Define the Banach spaces $\left(E,\|\cdot\|_{E}\right),\left(F,\|\cdot\|_{F}\right)$ by

$$
\begin{gathered}
E=\left\{x \in B^{\infty}: \sum_{j=1}^{\infty} \varepsilon_{j} x_{j} \text { converges in } L^{q}(B)\right\}, \\
\|x\|_{E}=E^{1 / q}\left\|\sum_{j=1}^{\infty} \varepsilon_{j} x_{j}\right\|^{q}
\end{gathered}
$$

and

$$
\begin{gathered}
F=\left\{x \in B^{\infty}: \sum_{j=1}^{n} j^{q}\left\|x_{j}\right\|^{q} / n^{q} \rightarrow 0\right\}, \\
\|x\|_{F}=\sup _{n}\left(\sum_{j=1}^{n} j^{q}\left\|x_{j}\right\|^{q} / n^{q}\right)^{1 / q} .
\end{gathered}
$$

Since the inclusion $E \subset F$ has a closed graph, there exists $C<\infty$ such that

$$
\sup _{n}\left(\sum_{j=1}^{n} j^{q}\left\|x_{j}\right\|^{q} / n^{q}\right)^{1 / q} \leq C\left(E\left\|\sum_{j=1}^{\infty} \varepsilon_{j} x_{j}\right\|^{q}\right)^{1 / q} .
$$

Thus for any $\left\{x_{j}\right\}_{j=1}^{n}$ in $B$ ( $C$ is a generic constant),

$$
\sum_{j=1}^{n} j^{q}\left\|x_{j}\right\|^{q} / n^{q} \leq C E\left\|\sum_{j=1}^{n} \varepsilon_{j} x_{j}\right\|^{q} .
$$


Fix $N \geq 1$ and let $y_{j}=0(1 \leq j \leq N)$ and $y_{j}=x_{j-N}(N<j \leq N+n)$, as in [4]. By (1),

$$
\sum_{j=1}^{n}(N+j)^{q}\left\|x_{j}\right\|^{q} \leq C(N+n)^{q} E\left\|\sum_{j=1}^{n} \varepsilon_{j} x_{j}\right\|^{q} .
$$

Choosing $N=n$ we see that

$$
(1 / 2)^{q} \sum_{j=1}^{n}\left\|x_{j}\right\|^{q} \leq C E\left\|\sum_{j=1}^{n} \varepsilon_{j} x_{j}\right\|^{q}
$$

proving that $B$ is of cotype $q$.

REMARK. A modification of the proof of Lemma 2.3 in [5] shows that if $\left\|X_{j}\right\| \leq j$ $(j \geq 1)$ and $\left\{X_{n}\right\} \in \mathrm{WLLN}$, then $E\left\|S_{n} / n\right\|^{q} \rightarrow 0$ for each $q \geq 1$. Thus the necessary condition in Theorem 1 may be replaced by $\sum_{j=1}^{n} E\left\|X_{j}\right\|^{q} / n^{q} \rightarrow 0$ if $\left\|X_{j}\right\| \leq j$.

EXAMPLE. Let $\phi_{n}$ be any sequence increasing to $+\infty$ and define

$$
X_{j}=\varepsilon_{j} j /\left(\phi_{j}\right)^{1 / 2 q} \quad\left(j=2^{n}, n=1,2, \ldots\right)
$$

and $X_{j}=0$ otherwise. Then $\left\{X_{n}\right\} \in \operatorname{SLLN}$ since $\left(S_{2^{n+1}}-S_{2^{n}}\right) / 2^{n} \rightarrow 0$ a.s. [10, p. 159], but $\phi_{2^{n}} / 2^{n q} \sum_{j \in I(n)}\left|X_{j}\right|^{q} \rightarrow \infty$ a.s. Thus the rate in Theorem 1 cannot be improved.

3. Sufficient conditions. Our results are again in terms of the validity of a real-valued SLLN. We do not place any restrictions on the magnitudes of the norms of the $X_{n}$ 's as in [5] or assume that $\left\{X_{n}\right\} \in$ WLLN. The latter hypothesis is needed in [5] even in the nonsymmetric case. To see this, consider for $p \in[1,2)$ the $l^{p}$ valued sequence $\left\{X_{n}\right\}_{n \geq 1}$ defined by

$$
X_{n}(\omega, k)=\varepsilon_{n}(\omega)\left(n /(L L n)^{2}\right)^{1 / 2} I_{\{n\}}(k) .
$$

$\left\{X_{n}\right\}$ satisfies the hypotheses of Theorem 2 in [5], but $\left\{X_{n}\right\} \notin$ SLLN, since for any $\omega$,

$$
\left\|\sum_{j=1}^{n} X_{j} / n\right\|=n^{-1}\left[\sum_{j=1}^{n}\left(j /(L L j)^{2}\right)^{p / 2}\right]^{1 / p} \geq C n^{-1}\left[n^{p / 2+1} /(L L n)^{p}\right]^{1 / p} \rightarrow \infty .
$$

The next lemma is crucial; it is implicit in [6].

LEMMA 1. Let $\left\{x_{n}\right\}$ be any sequence in a separable Banach space B. Set $S_{n}=$ $\sum_{j=1}^{n} \varepsilon_{j} x_{j}$ and $s_{n}^{2}=E\left\|S_{n}\right\|^{2}$. Then there exists $M=M(B)$ such that for each $\varepsilon>0$,

$$
P\left(\left\|S_{n}\right\| / s_{n}>\varepsilon\right) \leq 3 \exp \left(-\varepsilon^{2} / M^{2}\right)
$$

PROOF. We shall use a basic result of Kwapien [6]: the a.s. convergence of $\sum_{j=1}^{\infty} \varepsilon_{j} x_{j}$ implies that $E\left(\exp \alpha\left\|\sum_{j=1}^{\infty} \varepsilon_{j} x_{j}\right\|^{2}\right)<\infty$ for each $\alpha>0$. Define the 
Banach spaces $\left(E,\|\cdot\|_{E}\right)$ and $\left(F,\|\cdot\|_{F}\right)$ by

$$
\begin{gathered}
E=\left\{x \in B^{\infty}: \sum_{j=1}^{\infty} \varepsilon_{j} x_{j} \text { converges in } L^{2}(B)\right\} \\
\|x\|_{E}=\left(E\left\|\sum_{j=1}^{\infty} \varepsilon_{j} x_{j}\right\|^{2}\right)^{1 / 2} \text { and } \\
F=\left\{x \in B^{\infty}: E \exp \left(\alpha\left\|\sum_{j=1}^{\infty} \varepsilon_{j} x_{j}\right\|^{2}\right)<\infty \text { for each } \alpha>0\right\} \\
\|x\|_{F}=\inf \left\{t>0: E \exp \left(\left\|\sum_{j=1}^{\infty} \varepsilon_{j} x_{j}\right\|^{2} / t^{2}\right)\right\} \leq e .
\end{gathered}
$$

Kwapien's theorem asserts that $E \subset F$. By the closed graph theorem there exists $M<\infty$ such that

$$
\inf \left\{t>0: E \exp \left(\|S\|^{2} / t^{2}\right) \leq e\right\} \leq M\left(E\|S\|^{2}\right)^{1 / 2}
$$

where $S=\sum_{j=1}^{\infty} \varepsilon_{j} x_{j}$. In other words, for each $\delta>0, E \exp \left(\|S\|^{2} / M^{2} E\|S\|^{2}+\delta\right) \leq$ $e$, so on letting $\delta \rightarrow 0$,

$$
P\left(\left\|S_{n}\right\| / s_{n}>\varepsilon\right) \leq \exp \left(-\varepsilon^{2} / M^{2}\right) E \exp \left(\left\|S_{n}\right\|^{2} / M^{2} s_{n}^{2}\right) \leq 3 \exp \left(-\varepsilon^{2} / M^{2}\right),
$$

as required.

THEOREM 2. $B$ is of logtype $p(1 \leq p \leq 2)$ if and only if each sequence $\left\{X_{n}\right\}$ of independent symmetric $B$ valued random variables satisfying

$$
(L L n)^{p-1} / n^{p} \sum_{j=1}^{n}\left\|X_{j}\right\|^{p} \rightarrow 0 \quad \text { a.s. }
$$

also satisfies the SLLN.

PrOOF. Assume that $(L L n)^{p-1} / n^{p} \sum_{j=1}^{n}\left\|X_{j}\right\|^{p} \rightarrow 0$ a.s. and let $X_{n}=Y_{n}+Z_{n}$, where $Y_{n}=X_{j} I\left(\left\|X_{j}\right\| \leq j / L L j\right)$. Then $\left\{Y_{n}\right\}$ and $\left\{Z_{n}\right\}$ are both independent symmetric sequences. As before, $\left\{Z_{n}\right\} \in \operatorname{SLLN}$ iff $\sum_{j \in I(n)} Z_{j} / 2^{n} \rightarrow 0$ a.s. But

$$
\begin{aligned}
\left\|\sum_{j \in I(n)} Z_{j} / 2^{n}\right\| & \leq 1 / 2^{n} \sum_{j \in I(n)} I^{p-1}\left(\left\|X_{j}\right\|>j / L L j\right)\left\|X_{j}\right\| \\
& \leq C\left(L L 2^{n}\right)^{p-1} / 2^{n p} \sum_{j \in I(n)}\left\|X_{j}\right\|^{p} \rightarrow 0 \text { a.s. by assumption. }
\end{aligned}
$$

Next consider the sequence $\left\{Y_{n}\right\}$. Clearly $\left\{Y_{n}\right\} \in$ SLLN iff $\left\{\varepsilon_{n} Y_{n}(\omega)\right\} \in$ SLLN for almost all $\omega$. Choose an $\omega$ for which $(L L n)^{p-1} / n^{p} \sum_{j=1}^{n}\left\|x_{j}\right\|^{p} \rightarrow 0$, where $x_{n}=X_{n}(\omega)$. Let $y_{n}=Y_{n}(\omega)$. We shall show that the sequence $\left\{\varepsilon_{n} y_{n}\right\}$ satisfies 
the hypotheses of Theorem 2 in Kuelbs and Zinn [5] and thus satisfies the SLLN. It is evident that $\left\|\varepsilon_{n} y_{n}\right\| \leq n / L L n(n \geq 1)$. Also,

$$
\begin{aligned}
L L 2^{n} / 4^{n} \sum_{j \in I(n)}\left\|y_{j}\right\|^{2} & =L L 2^{n} / 2^{n p} \sum_{j \in I(n)}\left\|y_{j}\right\|^{p}\left\|y_{j}\right\|^{2-p} / 2^{n(2-p)} \\
& \leq C L L 2^{n} / 2^{n p} \cdot 1 /\left(L L 2^{n}\right)^{2-p} \sum_{j \in I(n)}\left\|y_{j}\right\|^{p} \\
& =C\left(L L 2^{n}\right)^{p-1} / 2^{n p} \sum_{j \in I(n)}\left\|y_{j}\right\|^{p} \\
& \leq C\left(L L 2^{n}\right)^{p-1} / 2^{n p} \sum_{j \in I(n)}\left\|x_{j}\right\|^{p} \rightarrow 0 .
\end{aligned}
$$

Hence,

$$
\sum_{n=1}^{\infty} \exp \left(-\varepsilon 4^{n}\right) /\left(\sum_{j \in I(n)}\left\|y_{j}\right\|^{2}\right) \leq C_{\varepsilon} \sum_{n=1}^{\infty} n^{-2}<\infty
$$

so that $\sum_{n=1}^{\infty} \exp (-\varepsilon / \Lambda(n))<\infty$ for each $\varepsilon>0$. We need to show that $\left\{\varepsilon_{n} y_{n}\right\} \in$ WLLN. Since $B$ is of logtype $p$,

$$
\begin{aligned}
P\left(\left\|\sum_{j=1}^{n} \varepsilon_{j} x_{j}\right\|>n \varepsilon\right) & \leq(n \varepsilon)^{-p} E\left\|\sum_{j=1}^{n} \varepsilon_{j} x_{j}\right\|^{p} \\
& \leq C(n \varepsilon)^{-p}(L L n)^{p-1} \sum_{j=1}^{n}\left\|x_{j}\right\|^{p} \rightarrow 0 .
\end{aligned}
$$

Thus $\left\{\varepsilon_{n} x_{n}\right\} \in \mathrm{WLLN}$. Since $(L L n)^{p-1} / n^{p} \sum_{j=1}^{n}\left\|x_{j}\right\|^{p} \rightarrow 0$,

$$
\left\|x_{n}\right\| \leq n /(L L n)^{(p-1) / p}
$$

if $n$ is large enough. By the remark in $\S 2,1 / n E\left\|\sum_{j=1}^{n} \varepsilon_{j} x_{j}\right\| \rightarrow 0$. The comparison principle (Lemma 4.1 in [3]) now shows that $1 / n E\left\|\sum_{j=1}^{n} \varepsilon_{j} y_{j}\right\| \rightarrow 0$. Thus $\left\{\varepsilon_{n} y_{n}\right\} \in \mathrm{WLLN}$.

Conversely, consider the Rademacher sequence $\left\{\varepsilon_{n} x_{n}\right\},\left\{x_{n}\right\} \subset B$. By hypothesis, $\left\{\varepsilon_{n} x_{n}\right\} \in \mathrm{SLLN}$ if $(L L n)^{p-1} / n^{p} \sum_{j=1}^{n}\left\|x_{j}\right\|^{p} \rightarrow 0$. Kronecker's lemma and an argument similar to the one in Theorem 1 now show that

$$
1 / n^{p} E\left\|\sum_{j=1}^{n} \varepsilon_{j} x_{j}\right\|^{p} \rightarrow 0 \text { if } \sum_{j=1}^{\infty}(L L j)^{p-1}\left\|x_{j}\right\|^{p} / j^{p}<\infty .
$$

Define the Banach spaces $\left(E,\|\cdot\|_{E}\right)$ and $\left(F,\|\cdot\|_{F}\right)$ by

$$
\begin{gathered}
E=\left\{x \in B^{\infty}: \sum_{j=1}^{\infty}(L L j)^{p-1}\left\|x_{j}\right\|^{p} / j^{p}<\infty\right\}, \\
\|x\|_{E}=\left[\sum_{j=1}^{\infty}(L L j)^{p-1}\left\|x_{j}\right\|^{p} / j^{p}\right]^{1 / p}
\end{gathered}
$$


and

$$
\begin{gathered}
F=\left\{x \in B^{\infty}: 1 / n^{p} E\left\|\sum_{j=1}^{n} \varepsilon_{j} x_{j}\right\|^{p} \rightarrow 0\right\}, \\
\|x\|_{F}=\sup _{n} 1 / n\left(E\left\|\sum_{j=1}^{n} \varepsilon_{j} x_{j}\right\|^{p}\right)^{1 / p} .
\end{gathered}
$$

Since the map $E \subset F$ has a closed graph, there exists $C<\infty$ such that

$$
\sup _{n} 1 / n\left(E\left\|\sum_{j=1}^{n} \varepsilon_{j} x_{j}\right\|^{p}\right)^{1 / p} \leq C\left(\sum_{j=1}^{\infty}(L L j)^{p-1}\left\|x_{j}\right\|^{p} / j^{p}\right)^{1 / p} .
$$

Hence

$$
E\left\|\sum_{j=1}^{n} \varepsilon_{j} x_{j}\right\|^{p} \leq C n^{p}\left(\sum_{j=1}^{n}(L L j)^{p-1}\left\|x_{j}\right\|^{p} / j^{p}\right) \quad(n \geq 1) .
$$

As in Theorem 1 , fix $N \geq 1$ and let $y_{j}=0(1 \leq j \leq N)$ and $y_{j}=x_{j-N}(N<j \leq$ $N+n)$. By (3),

$$
\begin{aligned}
E\left\|\sum_{j=1}^{n} \varepsilon_{j} x_{j}\right\|^{p} & \leq C(N+n)^{p}\left(\sum_{j=1}^{n}[L L(N+j)]^{p-1}\left\|x_{j}\right\|^{p} /(N+j)^{p}\right) \\
& \leq C\left\{(N+n)^{p}[L L(N+n)]^{p-1} /(N+1)^{p}\right\} \sum_{j=1}^{n}\left\|x_{j}\right\|^{p} .
\end{aligned}
$$

The fact that $B$ is of logtype $p$ follows on setting $N=n$.

EXAMPLES. Let $X_{j}=\varepsilon_{j} j /(L L j)^{3 / 4}\left(j=2^{n}, n=1,2, \ldots\right)$ and $X_{j}=0$ otherwise. Then

$$
\sum_{j=1}^{\infty} E\left|X_{j}\right|^{p} / j^{p}=\infty \quad \text { for each } p \in[1,2]
$$

so that the Hoffmann-Jørgensen and Pisier SLLN [4] is inconclusive. It is also easy to see that $\sum_{n=1}^{\infty} \Lambda(n, p)^{r}=\infty$ for each $p \in[1,2]$ and $r \in(0, \infty)(\Lambda(n, p)=$ $\left.1 / 2^{n p} \sum_{j \in I(n)} E\left\|X_{j}\right\|^{p}\right)$ so that Theorem 1 in Kuelbs and Zinn [5] is inconclusive as well. On the other hand, for any $p \in[1,2]$ and $n \in I(k)$,

$$
\begin{aligned}
(L L n)^{p-1} / n^{p} \sum_{j=1}^{n}\left|X_{j}\right|^{p} & \leq\left(L L 2^{k+1}\right)^{p-1} / 2^{k p} \sum_{j=1}^{2^{k+1}}\left|X_{j}\right|^{p} \\
& =\left(L L 2^{k+1}\right)^{p-1} / 2^{k p} \sum_{j=1}^{k+1} 2^{j p} /\left(L L 2^{j}\right)^{3 p / 4} \\
& \leq C\left(L L 2^{k}\right)^{p / 4-1} \rightarrow 0 \text { a.s. }
\end{aligned}
$$

so that $\left\{X_{n}\right\} \in \mathrm{SLLN}$.

Our second example shows that the rate in Theorem 2 is the best possible in the sense that for each $p \in[1,2]$, and for each sequence $\phi(n) \uparrow \infty$, there exists 
a sequence $\left\{X_{n}\right\}$ of independent symmetric real random variables satisfying the condition $\left[(L L n)^{p-1} / n^{p} \phi(n)\right] \sum_{j=1}^{n}\left|X_{j}\right|^{p} \rightarrow 0$ a.s. but failing the SLLN. Such a sequence may be defined by $X_{j}=\varepsilon_{j} 2^{n} / L L 2^{n}, j=2^{n}+1, \ldots, 2^{n}+\left[L L 2^{n}\right], n=$ $1,2, \ldots$, and $X_{j}=0$ otherwise. Then for any $k \in I(n)$ and $p \in[1,2]$,

$$
\begin{aligned}
& {\left[(L L k)^{p-1} / k^{p} \phi(k)\right] \sum_{j=1}^{k}\left|X_{j}\right|^{p}} \\
& \quad \leq\left[\left(L L 2^{n+1}\right)^{p-1} / 2^{n p} \phi\left(2^{n}\right)\right] \sum_{j=1}^{n+1}\left[L L 2^{j}\right] 2^{j p} /\left(L L 2^{j}\right)^{p} \\
& \quad \leq C\left(L L 2^{n+1}\right)^{p-1} / 2^{n p} \cdot 2^{(n+1) p} /\left(L L 2^{n+1}\right)^{p-1} \cdot 1 / \phi\left(2^{n}\right) \rightarrow 0 \quad \text { as } n \rightarrow \infty .
\end{aligned}
$$

On the other hand, we have $\Lambda(n)=1 / 4^{n} \cdot\left[L L 2^{n}\right] \cdot 4^{n} /\left(L L 2^{n}\right)^{2} \geq C / \log n$ so that $\sum_{n=1}^{\infty} \exp (-\varepsilon / \Lambda(n))=\infty$ for $\varepsilon$ small enough. Hence $\left\{X_{n}\right\} \notin \mathrm{SLLN}$, by Prokhorov's result [9].

COROLLARY. A sequence of independent symmetric random variables with values in a Hilbert space (or more generally a cotype 2, logtype 2 space) satisfies the $S L L N$ if $L L n / n^{2} \sum_{j=1}^{n}\left\|X_{j}\right\|^{2} \rightarrow 0$ a.s. and only if $1 / n^{2} \sum_{j=1}^{n}\left\|X_{j}\right\|^{2} \rightarrow 0$ a.s. Furthermore, the condition $\phi(n) / n^{2} \sum_{j=1}^{n}\left\|X_{j}\right\|^{2} \rightarrow 0$ a.s. is neither necessary nor sufficient for the $S L L N$ for any sequence $\phi(n) \uparrow \infty, \phi(n)=o(L L n)$.

We now ask when our conditions for the SLLN may be rephrased in terms of individual moments.

Proposition. Assume that $\left\|X_{n}\right\| \leq C n / L$ Ln a.s. $(n \geq 1)$ and $p \in[1,2]$. Then

$$
\begin{aligned}
& (L L n)^{p-1} / n^{p} \sum_{j=1}^{n}\left\|X_{j}\right\|^{p} \rightarrow 0 \quad \text { a.s. iff } \\
& {\left[(L L n)^{p-1} / n^{p}\right] \sum_{j=1}^{n}\left\|X_{j}\right\|^{P} \rightarrow 0 \quad \text { in probability }}
\end{aligned}
$$

and

(5)

$$
\sum_{n=1}^{\infty} \exp \left[-\varepsilon 2^{2 n p} /\left\{\left(L L 2^{n}\right)^{2 p-2} \sum_{j \in I(n)} E\left(\left\|X_{j}\right\|^{p}-\left\|X_{j}^{\prime}\right\|^{p}\right)^{2}\right\}\right]<\infty \quad(\varepsilon>0)
$$

where $X_{j}^{\prime}$ is an independent copy of $X_{j}(j \geq 1)$. (Here, (4) may be expressed in terms of the individual moments by the classical degenerate convergence criterion.)

PROOF. As mentioned in $\S 1,(L L n)^{p-1} / n^{p} \sum_{j=1}^{n}\left\|X_{j}\right\|^{p} \rightarrow 0$ a.s. if and only if (4) holds and

$$
(L L n)^{p-1} / n^{p} \sum_{j=1}^{n}\left(\left\|X_{j}\right\|^{p}-\left\|X_{j}^{\prime}\right\|^{p}\right) \rightarrow 0 \quad \text { a.s. }
$$

We have

$$
\left|\left\|X_{j}\right\|^{p}-\left\|X_{j}^{\prime}\right\|^{p}\right| \leq \frac{2 C j^{p}}{(L L j)^{p}} \leq \frac{C^{\prime} j^{p} /(L L j)^{p-1}}{L L\left(j^{p} /(L L j)^{p-1}\right)}
$$


so that we may apply a result of Loéve $[7$, p. 258] to conclude that (5) and (6) are equivalent. The assertion follows.

Notice that $E\left(\left\|X_{j}\right\|^{p}-\left\|X_{j}^{\prime}\right\|^{p}\right)^{2}=2 \operatorname{Var}\left(\left\|X_{j}\right\|^{p}\right) \leq 2 E\left\|X_{j}\right\|^{2 p}$, so that

$$
\begin{aligned}
& \left(L L 2^{n}\right)^{2 p-2} \sum_{j \in I(n)} E\left(\left\|X_{j}\right\|^{p}-\left\|X_{j}^{\prime}\right\|^{p}\right)^{2} / 2^{2 n p} \\
& \leq 2\left(L L 2^{n}\right)^{2 p-2} \sum_{j \in I(n)} E\left\|X_{j}\right\|^{2 p} / 2^{2 n p} \\
& \leq C\left(L L 2^{n}\right)^{2 p-2} \sum_{j \in I(n)} E\left\|X_{j}\right\|^{2}\left(2^{n} / L L 2^{n}\right)^{2 p-2} / 2^{2 n p}=C \Lambda(n, 2)
\end{aligned}
$$

so that (5) is a slight improvement of the Kuelbs-Zinn condition. On the other hand, (4) may do worse than their WLLN hypothesis.

ACKNOWLEDGMENT. I would like to thank my advisor, Professor Joel Zinn for his guidance and encouragement.

\section{REFERENCES}

1. A. Beck, D. P. Giesy, and P. Warren, Recent developments in the theory of strong law of large numbers for vector valued random variables, Theor. Probab. Appl. 20 (1975), 127-134.

2. A. P. Godbole, Dissertation, Michigan State University, East Lansing, Michigan, 1984.

3. J. Hoffmann-Jørgensen, Sums of independent Banach space valued random variables, Studia Math. 52 (1974), 159-186.

4. J. Hoffmann-Jørgensen and G. Pisier, The law of large numbers and the central limit theorem in Banach spaces, Ann. Probab. 4 (1976), 587-599.

5. J. Kuelbs and J. Zinn, Some stability results for vector valued random variables, Ann. Probab. 7 (1979), 75-84.

6. S. Kwapien, A theorem on the Rademacher series with vector coefficients, Probability in Banach Spaces, Lecture Notes in Math., vol. 526, Springer-Verlag, Berlin and New York, 1975, pp. 157-158.

7. M. Loeve, Probability theory (3rd ed.), Van Nostrand, Princeton, N. J., 1963.

8. S. V. Nagaev, On necessary and sufficient conditions for the strong law of large numbers, Theor. Probab. Appl. 17 (1972), 573-581.

9. Yu. V. Prokhorov, Some remarks on the strong law of large numbers, Theor. Probab. Appl. 4 (1959), 204-208.

10. W. F. Stout, Almost sure convergence, Academic Press, New York, 1974.

11. N. A. Volodin and S. V. Nagaev, A remark on the strong law of large numbers, Theor. Probab. Appl. 23 (1978), 810-813.

Department of Mathematical and Computer Sciences, Michigan TechnoLOGICAL UNIVERSITY, HOUGHTON, MICHIGAN 49931 